\title{
Mean-field instability of trapped dilute boson-fermion mixtures
}

\author{
R. Roth ${ }^{\text {f }}$ and H. Feldmeier \\ Gesellschaft für Schwerionenforschung (GSI), Planckstr. 1, 64291 Darmstadt, Germany
}

(Dated: October 24, 2018)

\begin{abstract}
The influence of boson-boson and boson-fermion interactions on the stability of a binary mixture of bosonic and fermionic atoms is investigated. The density profiles of the trapped mixture are obtained from direct numerical solution of a modified Gross-Pitaevskii equation that is selfconsistently coupled to the mean-field generated by the interaction with the fermionic species. The fermions which in turn feel the mean-field created by the bosons are treated in Thomas-Fermi approximation. We study the effects of different combinations of signs of the boson-boson and the boson-fermion scattering lengths and determine explicit expressions for critical particle numbers as function of these scattering lengths.
\end{abstract}

PACS numbers: 03.75.-b, 03.75.Fi, 32.80.Pj

Recent experimental successes in the trapping and cooling of mixtures of bosonic and fermionic atoms [1, 2, 3] constitute a new branch in the field of trapped ultracold gases. Similar to the purely bosonic gases bosonfermion mixtures offer unique possibilities to study fundamental quantum phenomena. Moreover they appear as a promising candidate to realize a BCS transition to a superfluid phase of the fermionic component 顿. One of the most appealing features of these systems is that the strength of the interaction between the atoms can be tuned in a wide range by utilizing a Feshbach resonance [5].

For the sympathetic cooling of a Fermi gas in binary boson-fermion mixtures the collapse caused by attractive interactions is responsible for a severe limitation of the lowest achievable temperature [1, 2]. The occurrence of a mean-field instability when the density or particle number exceeds a critical value was already studied experimentally for purely bosonic systems [6]. In this communication we investigate the interplay between boson-boson and boson-fermion interactions and the implications for the stability of the mixture.

In order to describe the properties of the binary bosonfermion mixture at zero temperature we first construct the energy functional in mean-field approximation. The many-body state $|\Psi\rangle$ of the mixed boson-fermion system is a direct product of a symmetric $N_{\mathrm{B}}$-body state $\left|\Psi_{\mathrm{B}}\right\rangle$ for the bosonic species and an antisymmetric $N_{\mathrm{F}}$-body state $\left|\Psi_{\mathrm{F}}\right\rangle$ for the fermions. The Hamiltonian of the interacting mixture reads

$$
\begin{aligned}
\boldsymbol{H} & =\sum_{i=1}^{N_{\mathrm{B}}}\left[\frac{\overrightarrow{\boldsymbol{p}}_{i}^{2}}{2 m_{\mathrm{B}}}+U_{\mathrm{B}}\left(\overrightarrow{\boldsymbol{x}}_{i}\right)\right]+\sum_{i=N_{\mathrm{B}}+1}^{N_{\mathrm{B}}+N_{\mathrm{F}}}\left[\frac{\overrightarrow{\boldsymbol{p}}_{i}^{2}}{2 m_{\mathrm{F}}}+U_{\mathrm{F}}\left(\overrightarrow{\boldsymbol{x}}_{i}\right)\right] \\
& +\sum_{i<j=1}^{N_{\mathrm{B}}} \frac{4 \pi a_{\mathrm{B}}}{m_{\mathrm{B}}} \delta^{(3)}\left(\boldsymbol{r}_{i j}\right)+\sum_{i=1}^{N_{\mathrm{B}}} \sum_{j=N_{\mathrm{B}}+1}^{N_{\mathrm{B}}+N_{\mathrm{F}}} \frac{4 \pi a_{\mathrm{BF}}}{m_{\mathrm{BF}}} \delta^{(3)}\left(\boldsymbol{r}_{i j}\right) .
\end{aligned}
$$

The first line contains the kinetic energy operators and the external trapping potentials $U_{\mathrm{B}}(\vec{x})$ and $U_{\mathrm{F}}(\vec{x})$ for the bosonic and the fermionic species with masses $m_{\mathrm{B}}$ and $m_{\mathrm{F}}$, respectively.
Due to the large average distance the atom-atom interaction can in general be described by an effective contact interaction for all partial waves [7, 8]. In a binary boson-fermion mixture one has to distinguish three interaction types: boson-boson interactions, boson-fermion interactions, and fermion-fermion interactions. The swave interaction between two bosons is described by the third term of Eq. (11), where $a_{\mathrm{B}}$ is the s-wave scattering length. Since we consider a pure Bose-Einstein condensate at zero temperature only the s-wave term is needed. Higher even partial waves are negligible. For the interaction between a bosonic and a fermionic atom s- and p-wave terms contribute. The operator of the s-wave boson-fermion contact interaction forms the last term of the Hamiltonian (11), where $a_{\mathrm{BF}}$ is the corresponding swave scattering length and $m_{\mathrm{BF}}=2 m_{\mathrm{B}} m_{\mathrm{F}} /\left(m_{\mathrm{B}}+m_{\mathrm{F}}\right)$ is twice the reduced mass of the pair. Since the s-wave interaction dominates in many cases of interest, e.g. the ${ }^{6} \mathrm{Li} /{ }^{7} \mathrm{Li}$ mixture, we will neglect the p-wave interaction for this discussion. For identical fermions s-wave contact interactions are prohibited by the Pauli principle. The first nonvanishing contribution is the nonlocal p-wave interaction, which will also be neglected in the following. However, p-wave interactions can have significant influence on the structure and stability of the fermionic component as we discussed earlier [7, 9].

The expectation value of the Hamiltonian (1) calculated with the many-body state $|\Psi\rangle=\left|\Psi_{\mathrm{B}}\right\rangle \otimes\left|\Psi_{\mathrm{F}}\right\rangle$, defines the energy density of the mixture

$$
E=\langle\Psi|\boldsymbol{H}| \Psi\rangle=\int \mathrm{d}^{3} x \mathcal{E}\left[n_{\mathrm{B}}, n_{\mathrm{F}}\right](\vec{x}) .
$$

The energy density $\mathcal{E}\left[n_{\mathrm{B}}, n_{\mathrm{F}}\right]$ is decomposed into a purely bosonic part (B), a fermionic part (F) and the interaction part between the two species $(\mathrm{BF})$

$$
\mathcal{E}\left[n_{\mathrm{B}}, n_{\mathrm{F}}\right]=\mathcal{E}_{\mathrm{B}}\left[n_{\mathrm{B}}\right]+\mathcal{E}_{\mathrm{F}}\left[n_{\mathrm{F}}\right]+\mathcal{E}_{\mathrm{BF}}\left[n_{\mathrm{B}}, n_{\mathrm{F}}\right] .
$$

For the calculation of $\mathcal{E}_{\mathrm{B}}\left[n_{\mathrm{B}}\right]$ we assume that the bosons are in a pure Bose-Einstein condensate, i.e. the $N_{\mathrm{B}}$-boson state $\left|\Psi_{\mathrm{B}}\right\rangle$ is given by a direct product of identical single particle states $\left|\phi_{\mathrm{B}}\right\rangle$. This immediately leads 


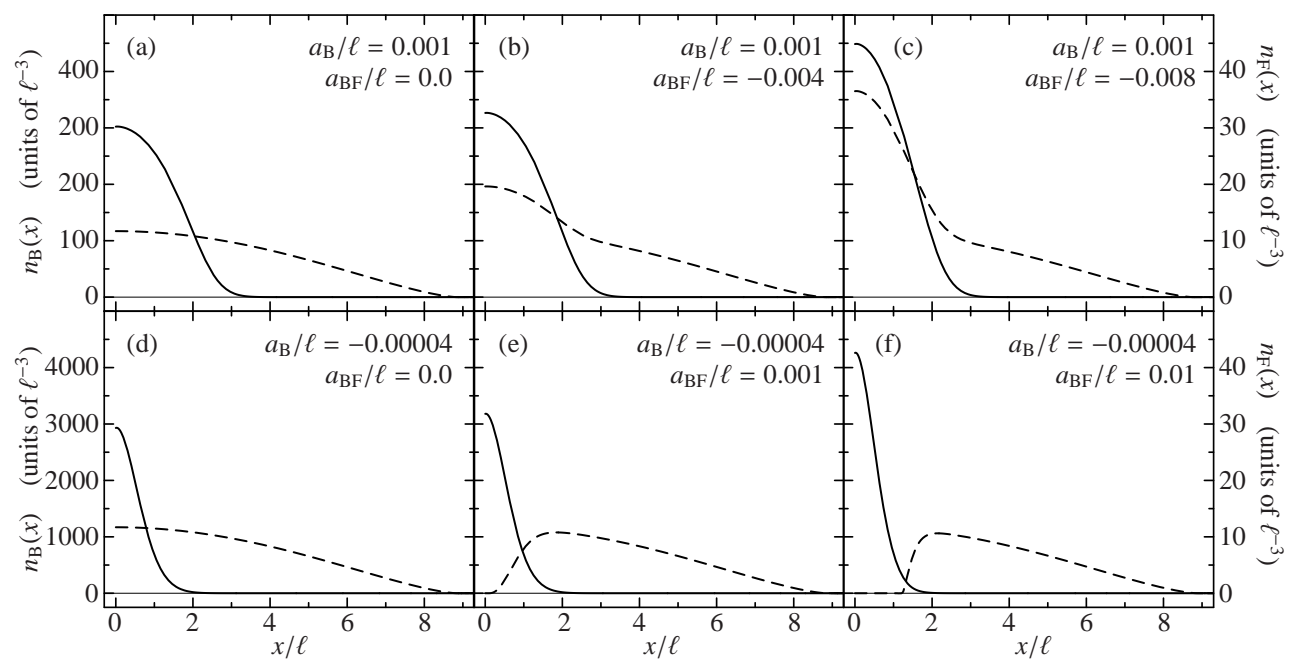

FIG. 1: Radial density profiles of a boson-fermion mixture with $N_{\mathrm{F}}=N_{\mathrm{B}}=10000$ for different interaction strengths. The boson density $n_{\mathrm{B}}(\vec{x})$ is given by the solid line (left scale) and the fermion density $n_{\mathrm{F}}(\vec{x})$ by the dashed line (right scale). The upper row shows examples with increasing bosonfermion attraction and fixed $a_{\mathrm{B}} / \ell=0.001$. The lower row depicts examples with increasing boson-fermion repulsion and $a_{\mathrm{B}} / \ell=-0.00004$. to the standard Gross-Pitaevskii energy density [10]

$$
\begin{aligned}
\mathcal{E}_{\mathrm{B}}\left[n_{\mathrm{B}}\right](\vec{x}) & =U_{\mathrm{B}}(\vec{x}) n_{\mathrm{B}}(\vec{x})+\frac{1}{2 m_{\mathrm{B}}}\left|\vec{\nabla} n_{\mathrm{B}}^{1 / 2}(\vec{x})\right|^{2} \\
& +\frac{2 \pi a_{\mathrm{B}}}{m_{\mathrm{B}}} n_{\mathrm{B}}^{2}(\vec{x}),
\end{aligned}
$$

where $n_{\mathrm{B}}(\vec{x})=\Phi_{\mathrm{B}}^{2}(\vec{x})=N_{\mathrm{B}}\left\langle\vec{x} \mid \phi_{\mathrm{B}}\right\rangle^{2}$ is the ground-state boson density.

For the evaluation of the parts of the energy density that involve the fermionic species we employ the ThomasFermi approximation. It was shown that this is an excellent approximation for the particle numbers considered here [11]. The fermionic part of the energy density in Thomas-Fermi approximation reads [7]

$$
\mathcal{E}_{\mathrm{F}}\left[n_{\mathrm{F}}\right](\vec{x})=U_{\mathrm{F}}(\vec{x}) n_{\mathrm{F}}(\vec{x})+\frac{3^{5 / 3} \pi^{4 / 3}}{52^{1 / 3} m_{\mathrm{F}}} n_{\mathrm{F}}^{5 / 3}(\vec{x}) .
$$

The interaction between the bosonic and the fermionic species yields the contribution

$$
\mathcal{E}_{\mathrm{BF}}\left[n_{\mathrm{B}}, n_{\mathrm{F}}\right](\vec{x})=\frac{4 \pi a_{\mathrm{BF}}}{m_{\mathrm{BF}}} n_{\mathrm{B}}(\vec{x}) n_{\mathrm{F}}(\vec{x})
$$

The functional minimization of the total energy (2) under the constraint of given numbers of bosons and fermions leads to the density profiles of the trapped gas. The constraints are implemented by introducing the chemical potentials $\mu_{\mathrm{B}}$ and $\mu_{\mathrm{F}}$ and minimizing the transformed energy

$$
F=\int \mathrm{d}^{3} x\left[\mathcal{E}\left[n_{\mathrm{B}}, n_{\mathrm{F}}\right](\vec{x})-\mu_{\mathrm{B}} n_{\mathrm{B}}(\vec{x})-\mu_{\mathrm{F}} n_{\mathrm{F}}(\vec{x})\right] .
$$

Variation with respect to the fermion density $n_{\mathrm{F}}(\vec{x})$ immediately leads to an equation for the density distribution

$$
n_{\mathrm{F}}(\vec{x})=\frac{\sqrt{2 m_{\mathrm{F}}^{3}}}{3 \pi^{2}}\left[\mu_{\mathrm{F}}-U_{\mathrm{F}}(\vec{x})-\frac{4 \pi a_{\mathrm{BF}}}{m_{\mathrm{BF}}} n_{\mathrm{B}}(\vec{x})\right]^{3 / 2} .
$$

From the functional variation of the energy (7) with respect to the bosonic density we obtain an Euler-Lagrange differential equation for $\Phi_{\mathrm{B}}(\vec{x})=\sqrt{n_{\mathrm{B}}(\vec{x})}$

$$
\begin{aligned}
{\left[-\frac{1}{2 m_{\mathrm{B}}} \vec{\nabla}^{2}\right.} & +U_{\mathrm{B}}(\vec{x})+\frac{4 \pi a_{\mathrm{BF}}}{m_{\mathrm{BF}}} n_{\mathrm{F}}(\vec{x}) \\
& \left.+\frac{4 \pi a_{\mathrm{B}}}{m_{\mathrm{B}}} \Phi_{\mathrm{B}}^{2}(\vec{x})\right] \Phi_{\mathrm{B}}(\vec{x})=\mu_{\mathrm{B}} \Phi_{\mathrm{B}}(\vec{x}) .
\end{aligned}
$$

This is a modified Gross-Pitaevskii equation which includes the mean-field contribution generated by the interaction with the fermionic species.

The simultaneous solution of the coupled Eqs. (8) and (9) gives the density profiles of the two species. We solve the nonlinear differential equation (9) with an efficient quantum diffusion algorithm using a fast Fourier transformation [12]. The ground state solution is extracted by successive application of the time evolution operator for a small imaginary time step. We accomplish the solution of the coupled problem by a simple iterative procedure: (0) the boson density is initialized with the Gaussian profile of the noninteracting Bose gas with $N_{\mathrm{B}}$ particles. (1) The fermion density is calculated using (8) with $\mu_{\mathrm{F}}$ adjusted such that the integral over $n_{\mathrm{F}}(\vec{x})$ gives the desired particle number $N_{\mathrm{F}}$. (2) A single imaginary time-step is performed using the mean-field according to the boson and fermion densities obtained in the previous two steps. The resulting $n_{\mathrm{B}}(\vec{x})$ is normalized to $N_{\mathrm{B}}$ and used as initialization for the next iteration cycle.

With these tools we investigate the instability of the boson-fermion mixture against collapse induced by attractive boson-boson or fermion-boson interactions. In order to keep the discussion simple we restrict ourselves to spherical symmetric systems with equal numbers of bosons and fermions $N_{\mathrm{B}}=N_{\mathrm{F}}$. We assume equal masses for the two species $m=m_{\mathrm{B}}=m_{\mathrm{F}}=m_{\mathrm{BF}}$ and identical parabolic trapping potentials $U_{\mathrm{B}}(x)=U_{\mathrm{F}}(x)=$ $x^{2} /\left(2 m \ell^{4}\right)$. The oscillator length $\ell=(m \omega)^{-1 / 2}$ serves as fundamental length unit for the numerical treatment. A more general treatment including asymmetric boson- 
fermion mixtures in deformed traps will be presented in a subsequent paper.

First we consider the case of repulsive boson-boson and attractive boson-fermion interactions $\left(a_{\mathrm{B}} \geq 0, a_{\mathrm{BF}}<0\right)$. Here the attractive interaction between the species induces a mean-field collapse if the densities or particle numbers exceed a critical value. The upper row of Fig. 11 shows the density profiles of (meta)stable configurations with $N_{\mathrm{B}}=N_{\mathrm{F}}=10^{4}$ particles for three different values of the boson-fermion scattering length $a_{\mathrm{BF}}$ and fixed $a_{\mathrm{B}} / \ell=0.001$ (corresponds to $a_{\mathrm{B}} \approx 20 a_{\mathrm{Bohr}}$ for a typical trap with $\ell=1 \mu \mathrm{m}$ ). Due to the Pauli principle the fermionic density distribution is much more spread out and has a significantly lower central density than the bosonic distribution with the same particle number (notice the different scales for $n_{\mathrm{B}}(\vec{x})$ and $n_{\mathrm{F}}(\vec{x})$ in Fig. 1). Attractive boson-fermion interactions generate an attractive mean-field for bosons proportional to the density of the fermions and vice versa. This causes an increase of both densities in the overlap region as can be seen in panel (b) of Fig. 1. With increasing strength of the boson-fermion attraction the fermion density grows substantially. As shown in Fig. 11(c) the fermion density can easily be increased by a factor 3 compared to the noninteracting case.

If the strength of the boson-fermion interaction exceeds a critical value then the mixture collapses towards high densities. In this case the attractive mean-field is not stabilized by the positive kinetic energy contribution or the repulsive boson-boson interaction any more, i.e. the gas can lower its energy by contracting and increasing the density in the central region. This phenomenon was investigated in detail for purely bosonic [13] and purely fermionic [7, 9] systems. To our knowledge the collapse of boson-fermion mixtures was studied only inchoately using parameterized density profiles 14.

We can determine the critical boson-fermion scattering length $a_{\mathrm{BF}}$ for which the collapse occurs with a rather simple numerical procedure. The quantum diffusion algorithm used to obtain the solution of the Gross-Pitaevskii equation (9) diverges if the mean-field instability occurs, i.e., the change of the boson density in the trap center increases for successive imaginary time steps. Thus by observing the convergence behavior of the central density during the imaginary time evolution we can decide whether the mixture is stable or collapses.

To obtain a simple measure for the stability we proceed in two steps: First we determine numerically the critical boson-fermion scattering length for a set of particle numbers $N_{\mathrm{F}}=N_{\mathrm{B}}=500 \ldots 10^{6}$ and boson-boson scattering lengths $a_{\mathrm{B}} / \ell=0 \ldots 0.003$. Then we fit a parametrization which connects the particle number with the two scattering lengths to this data set. This leads to an expression for the critical particle number $N_{\text {cr }}$ as function of the scattering lengths $a_{\mathrm{B}} / \ell \geq 0$ and $a_{\mathrm{BF}} / \ell<0$ :

$$
N_{\mathrm{cr}}\left(a_{\mathrm{B}}, a_{\mathrm{BF}}, \ell\right)=\frac{0.283}{\left|a_{\mathrm{BF}} / \ell\right|^{1.78}}+\frac{0.374\left(a_{\mathrm{B}} / \ell\right)^{2.36}}{\left|a_{\mathrm{BF}} / \ell\right|^{5.69}} .
$$

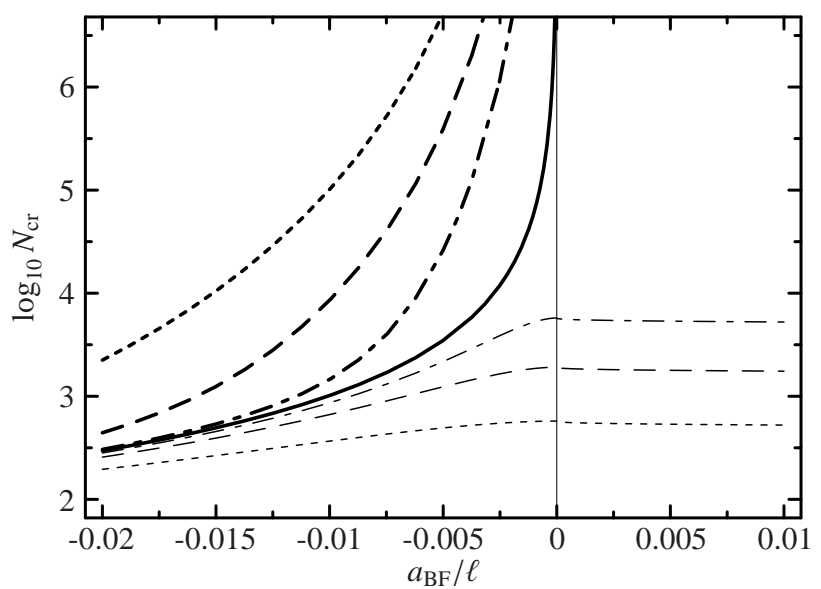

FIG. 2: Logarithm of the critical particle number as function of the boson-fermion scattering length $a_{\mathrm{BF}} / \ell$ for different values of the boson-boson scattering length. The solid line corresponds to $a_{\mathrm{B}} / \ell=0$. Thick lines show the behavior for repulsive boson-boson interactions: $a_{\mathrm{B}} / \ell=0.0003$ (thick dash-dotted), 0.001 (thick dashed), 0.003 (thick dotted). Thin lines correspond to boson-boson attraction: $a_{\mathrm{B}} / \ell=-0.0001$ (thin dash-dotted), -0.0003 (thin dashed), -0.001 (thin dotted).

Any mixture with $N_{\mathrm{B}}=N_{\mathrm{F}}>N_{\text {cr }}$ is unstable against mean-field induced collapse.

Figure 2 depicts the critical particle number as function of the boson-fermion scattering length $a_{\mathrm{BF}}$ for different values of the boson-boson scattering length $a_{\mathrm{B}}$. The thick lines show the critical particle number obtained from (10) for repulsive boson-boson interactions. Notice that the values of $a_{\mathrm{B}} / \ell$ used in the plot are typically one order of magnitude smaller than the range shown for $a_{\mathrm{BF}} / \ell$; this emphasizes the strong influence of the bosonboson interaction.

We observe that a moderate boson-fermion attraction causes a severe limitation of the particle number of the (meta)stable mixture. For $a_{\mathrm{B}} / \ell=0$ and $a_{\mathrm{BF}} / \ell=-0.01$, which corresponds to $a_{\mathrm{BF}} \approx-200 a_{\mathrm{Bohr}}$ for $\ell=1 \mu \mathrm{m}$, the mixture is stable only up to $N_{\mathrm{B}}=N_{\mathrm{F}} \approx 1000$. The inclusion of a repulsive boson-boson interaction leads to a significant stabilization, i.e. an increase of the critical particle number. For the above example, the critical particle number grows from $N_{\mathrm{cr}} \approx 1000$ to 8500 if a weak boson-boson repulsion with $a_{\mathrm{B}} / \ell=0.001$ is included.

As a second class of systems we consider mixtures where both, the boson-fermion and the boson-boson interaction are attractive $\left(a_{\mathrm{B}}<0, a_{\mathrm{BF}}<0\right)$. Compared to the previous class the attractive boson-boson interaction enhances the attractive mean-field generated by the boson-fermion interaction and abets the instability. The critical particle numbers are significantly reduced.

We obtain a relation between the scattering lengths $a_{\mathrm{B}}$ and $a_{\mathrm{BF}}$ and the critical particle number in the same way as before. The resulting parametrization of the critical 
particle number for $a_{\mathrm{B}}<0$ and $a_{\mathrm{BF}}<0$ reads

$$
N_{\mathrm{cr}}\left(a_{\mathrm{B}}, a_{\mathrm{BF}}, \ell\right)=\frac{0.575}{\left|a_{\mathrm{B}} / \ell\right|+2.03\left|a_{\mathrm{BF}} / \ell\right|^{1.78}} .
$$

For $a_{\mathrm{BF}}=0$ we recover the well known relation between the critical particle number and $\left|a_{\mathrm{B}}\right|$ for a pure Bose gas [10, 13]. The additional term in the denominator describes the modification of $N_{\mathrm{cr}}$ in the presence of an attractive boson-fermion interaction. In the limiting case $a_{\mathrm{B}}=0$ this relation coincides with the corresponding limit of (10).

The thin curves in Fig. 2 show the behavior of the critical particle number for $a_{\mathrm{B}} / \ell<0$. The presence of the attractive boson-boson interaction reduces the critical particle number for $a_{\mathrm{BF}}<0$ significantly. With increasing strength of the boson-boson attraction the influence of the boson-fermion attraction is largely reduced.

Finally we consider mixtures with attractive bosonboson and repulsive boson-fermion interactions $\left(a_{\mathrm{B}}<\right.$ $\left.0, a_{\mathrm{BF}} \geq 0\right)$. The ${ }^{6} \mathrm{Li} /{ }^{7} \mathrm{Li}$ mixture used in the experiment of Truscott et al. [1] belongs to this class of interactions. The lower row of Fig. 1 shows the density profiles for three different values of $a_{\mathrm{BF}} \geq 0$. Already for a very weak boson-fermion repulsion the two species separate spatially [see Fig. 1](e)], the bosons occupy the central region of the trap (boson core) and the fermions constitute a shell around it [11, 15]. This structure may have interesting implications for the mean-field instability of the bosons: The fermionic shell compresses the boson core, i.e. increases the maximum boson density as it is clearly seen in Fig. 1 (f). This could promote the meanfield collapse in the presence of attractive boson-boson interactions and lower the critical particle number.

The dependence of $N_{\mathrm{cr}}$ on the scattering lengths, which is obtained from the direct numerical solution of the cou- pled problem, is for $a_{\mathrm{B}}<0$ and $a_{\mathrm{BF}} \geq 0$ very well described by the parametrization

$$
N_{\mathrm{cr}}\left(a_{\mathrm{B}}, a_{\mathrm{BF}}, \ell\right)=\frac{0.575-0.230\left(a_{\mathrm{BF}} / \ell\right)^{0.333}}{\left|a_{\mathrm{B}} / \ell\right|} .
$$

The thin curves for $a_{\mathrm{BF}} / \ell>0$ in Fig. 2 show this dependence. Obviously the influence of the repulsive boson-fermion interaction on the critical particle number is marginal. The critical particle number reduces slightly if $a_{\mathrm{BF}} / \ell$ is increased. This can be attributed to the compression of the boson core mentioned before. Although the boson-fermion interaction has a strong influence on the density profiles, its influence on the critical particle number is negligible.

In summary we have investigated the mean-field instability of binary boson-fermion mixtures with equal particle numbers. We solved the coupled Gross-Pitaevskii equation numerically and obtained the critical particle number as function of the boson-boson and the bosonfermion scattering lengths. We have shown that the boson-boson and the boson-fermion interaction have very different effects on the collapse: In the presence of attractive boson-fermion interactions $\left(a_{\mathrm{BF}}<0\right)$ the system is stabilized by weak repulsive and destabilized by weak attractive boson-boson interactions. In contrast repulsive boson-fermion interactions $\left(a_{\mathrm{BF}}>0\right)$ cannot stabilize the mixture against collapse due to attractive boson-boson interactions. With regard to the recent experiments using mixtures of ${ }^{6} \mathrm{Li}\left(F=3 / 2, m_{F}=3 / 2\right)$ and ${ }^{7} \mathrm{Li}\left(F=2, m_{F}=2\right)$ 过, 2] this implies that the collapse due to the attractive boson-boson interaction is influenced only marginally by the boson-fermion repulsion.
* Electronic address: r.roth@gsi.de

$\dagger$ Electronic address: h.feldmeier@gsi.de

[1] A. G. Truscott, K. E. Strecker, W. I. McAlexander, G. Partridge, and R. Hulet, Science 291, 2570 (2001).

[2] F. Schreck, L. Khaykovich, K. L. Corwin, G. Ferrari, T. Bourdel, J. Cubizolles, and C. Salomon, Phys. Rev. Lett. 87, 080403 (2001).

[3] J. Goldwin, S. B. Papp, B. DeMarco, and D. S. Jin (2001), cond-mat/0108287.

[4] M. Houbiers, R. Ferwerda, H. T. C. Stoof, W. I. McAlexander, C. A. Sackett, and R. G. Hulet, Phys. Rev. A 56, 4864 (1997).

[5] M. Houbiers, H. T. C. Stoof, W. I. McAlexander, and R. G. Hulet, Phys. Rev. A 57, R1497 (1998).

[6] E. A. Donley, N. R. Claussen, S. L. Cornish, J. L. Roberts, E. A. Cornell, and C. E. Wieman, Nature 412, 295 (2001).
[7] R. Roth and H. Feldmeier, to be published in Phys. Rev. A 64 (2001), cond-mat/0102416.

[8] R. Roth and H. Feldmeier, J. Phys. B 33, L787 (2000).

[9] R. Roth and H. Feldmeier, to be published in J. Phys. B 34 (2001), cond-mat/0108255.

[10] F. Dalfovo, S. Giorgini, L. P. Pitaevskii, and S. Stringari, Rev. Mod. Phys. 71, 463 (1999).

[11] N. Nygaard and K. Mølmer, Phys. Rev. A 59, 2974 (1999).

[12] M. F. Feagin, Quantum Methods with Mathematica (Springer-Verlag, New York, 1994).

[13] A. Eleftheriou and K. Huang, Phys. Rev. A 61, 043601 (2000).

[14] T. Miyakawa, T. Suzuki, and H. Yabu, Phys. Rev. A 64, 033611 (2001).

[15] K. Mølmer, Phys. Rev. Lett. 80, 1804 (1998). 